\title{
Test of Purging a small tank with Argon
}

\author{
W. Jaskierny, H. Jostlein, S. Pordes, P.A. Rapidis,' T. Tope \\ Particle Physics Division, Fermilab
}

October 28, 2006

\section{Introduction}

One of the challenges facing our large liquid argon TPC is to remove the atmosphere of air to the point where the remaining oxygen can be removed by chemical or filtration techniques economically and in a reasonable time. This note describes a simple demonstration of purging the tank with argon gas inserted smoothly at the bottom of the tank to achieve concentrations of oxygen in the few 10's of ppm level. The purging is well described by an analytic diffusion model. The number of volume changes required to reach $100 \mathrm{ppm}$ is a factor of 3 fewer than predicted in a perfect mixing model.

A stainless steel tank of diameter $5 \mathrm{ft}$ and height $8.25 \mathrm{ft}$, previously used to store oil for the Miniboone experiment, was power washed and the hatch sealed. Two oxygen sensors of the type used in the Tevatron oxygen monitoring system [1] were hung at heights of $2 \mathrm{ft}$ and $6 \mathrm{ft}$ inside the tank, and a $0-5000 \mathrm{ppm}$ oxygen analyser [2] sampled the outlet gas from the top of the tank. The first figure is a photograph of the tank and a schematic showing the location of the oxygen sensors. The dashed boxes are intended to suggest the diffusion of the boundary between argon and air as the purge develops. The inlet diffuser was made from four 3 inch OD, 6 inch long cartridge air-filters stuck end to end. The flow was set to produce a vertical rate of $3.68 \mathrm{ft} / \mathrm{hr}$, a rate considered appropriate for a large tank.

The next figure shows an overview of the data from the two oxygen sensors and the oxygen analyser. Note that the analyser data refer to the right hand scale. Each sensor sees a well defined transition from air to argon and after 6 hrs or 2.7 volume changes the oxygen concentration at the top of the tank has fallen to $100 \mathrm{ppm}$. This is a reduction in oxygen concentration by a factor of 2000 ; in a perfect mixing model this would require 7.6 volume changes.

The third figure shows a comparison of an analytic calculation (the formula is in the figure) of perfect flow accompanied by diffusion - the diffusion coefficient is given in [3]. The agreement is quite satisfactory.

\footnotetext{
${ }^{*}$ now at Demokritos Institute, Athens, Greece
} 

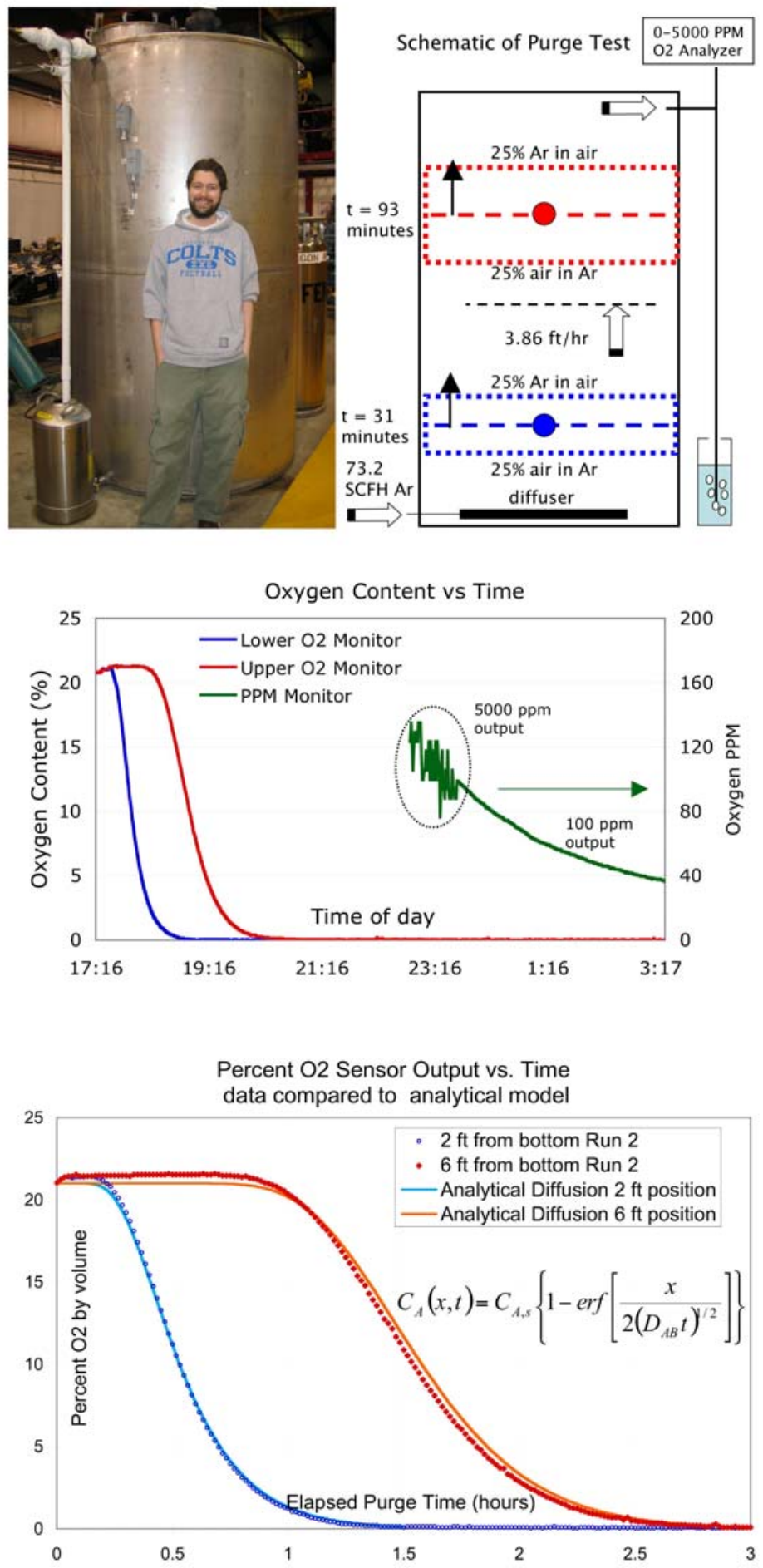
The final figure shows what happens when Nitrogen is introduced at the bottom of the tank. As can be seen, the sensors at different levels have similar readings and the mixing is indeed close to perfect.

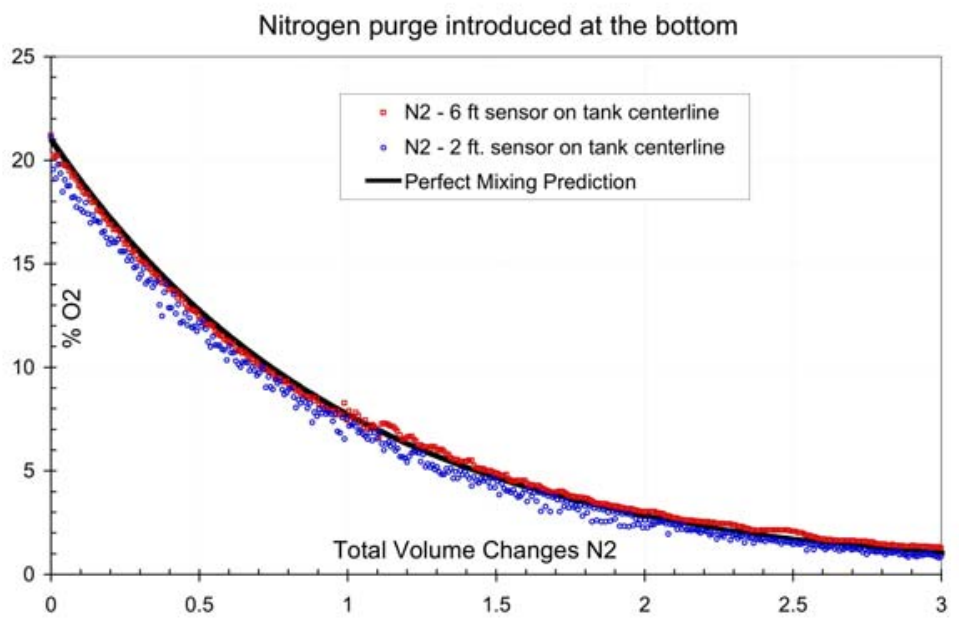

\section{References}

[1] See 'Electrical schematic for AD O2 cell preamp' in http://lartpc-docdb.fnal.gov/cgi-bin/ShowDocument?docid=88

[2] Delta F 310, Delta F Corporation, Woburn, MA.

[3] Table 40 "Experimental Gas Ordinary Diffusion Coefficients at One Atmosphere" for Nitrogen-Argon on page 2-94 of the "Handbook of Heat Transfer" Edited by Warren M. Rohsenow and James P. Hartnett. 